\title{
ER Dilation and Aberrant Zymogen Granule Phenotype in Secretory Cells of ERdj4 -/- Mice
}

\author{
C.-L. Na, ${ }^{*}$ M. Dong*, and T. E.Weaver* \\ *: Division of Pulmonary Biology, Cincinnati Children's Hospital Medical Center, Cincinnati, OH \\ 45229
}

The ER resident protein ERdj4 is part of the protein quality control machinery that participates in ER associated degradation. ERdj4 expression was elevated in tansfected HEK 293 cells expressing mutant pulmonary surfactant protein C (SP-C). Knockdown of ERdj4 inhibited mutant SP-C degradation in XBP-1 deficient transfected mouse embryonic fibroblasts (MEFs), while degradation of mutant SP-C was restored after transient expression of wild type (WT) ERdj4. Although ERdj4 is essential for recognition and degradation of mutant proteins, it is not clear if loss of ERdj4 function alone is sufficient to induce ER stress in vivo. To answer this question, ERdj4 -/- MEFs, submandibular glands, pancreas, and kidney, obtained from WT and ERdj4 gene trap (ERdj4 -/-) mice, were chosen for EM analyses to determine whether loss of ERdj4 induces any ER stress phenotypes.

Ultrastructural analyses demonstrated that ERdj4 -/- MEFs had different morphology compared to WT MEFs. Mild ER dilation was detected in ERdj4 -/- MEFs as well as in salivary exocrine cells of ERdj4 -/- mice. Severe ER dilation occurred in some ERdj4 -/- MEFs that had large inclusions near Golgi complex (Fig. 1). Similar inclusions were also detected in the salivary glands of ERdj4 -/mice (not shown).

Pancreatic exocrine cells of ERdj4 -/- mice had mild ER cisternae dilation and aberrant zymogen granules. Electron dense zymogen granules and immature zymogen granules (Fig. 2) similar to neuroendocrine dense core granules of pancreatic tumor cells were colocalized to pancreatic exocrine cells. In contrast, only electron dense zymogen granules were found in WT exocrine cells. Large inclusions similar to those detected in ERdj4 -/- MEFs were detected in some ERdj4 -/pancreatic exocrine cells (not shown). Histological examination by both light and electron microscopy determined that the immature zymogen granule phenotype was not tumor related, but a novel phenotype likely resulting from knockdown of ERdj4 expression.

In the convoluted tubules of ERdj4 -/- mice, ER dilation was detected in epithelial cells as well as in interstitial cells. These epithelial cells had severe ER and vesicle dilation localized to the apical cytoplasm, perinulear region (Fig. 3), and basal ER network, while microvilli of these cells had normal morphology comparable to WT epithelial cells. Golgi dilation similar to that detected in Erdj4 -/- MEFs and salivary glands was present in some epithelial cells. Focal thickening of basal membrane was detected in some convoluted tubules, however, collagen and smooth muscle infiltration into basal membrane was minimal (not shown).

These studies demonstrate that loss of ERdj4 alone is sufficient to induce ER dilation in epithelial cells. Detection of dilated ER, Golgi and inclusions in ERdj4 -/- mice suggests that ERdj4 may have tissue specific functions other than recognition and degradation of mutant proteins. Supported by NIH HL086492 (TEW). 

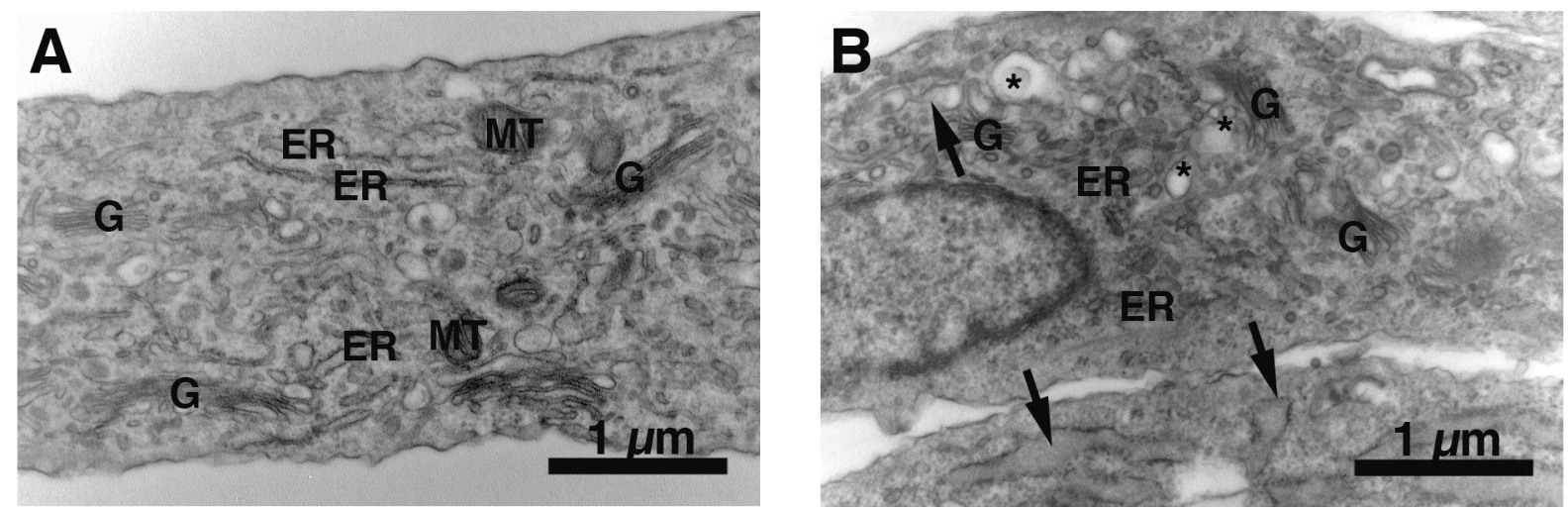

Fig 1. MEFs isolated from (A) WT or (B) ERdj4 -/- mice. Note that dilated ER (arrows) and large inclusions (*) near Golgi complex were detected in MEFs of ERdj4 -/- mice. ER: endoplasmic reticulum; G: Golgi complex; MT: mitochondria.
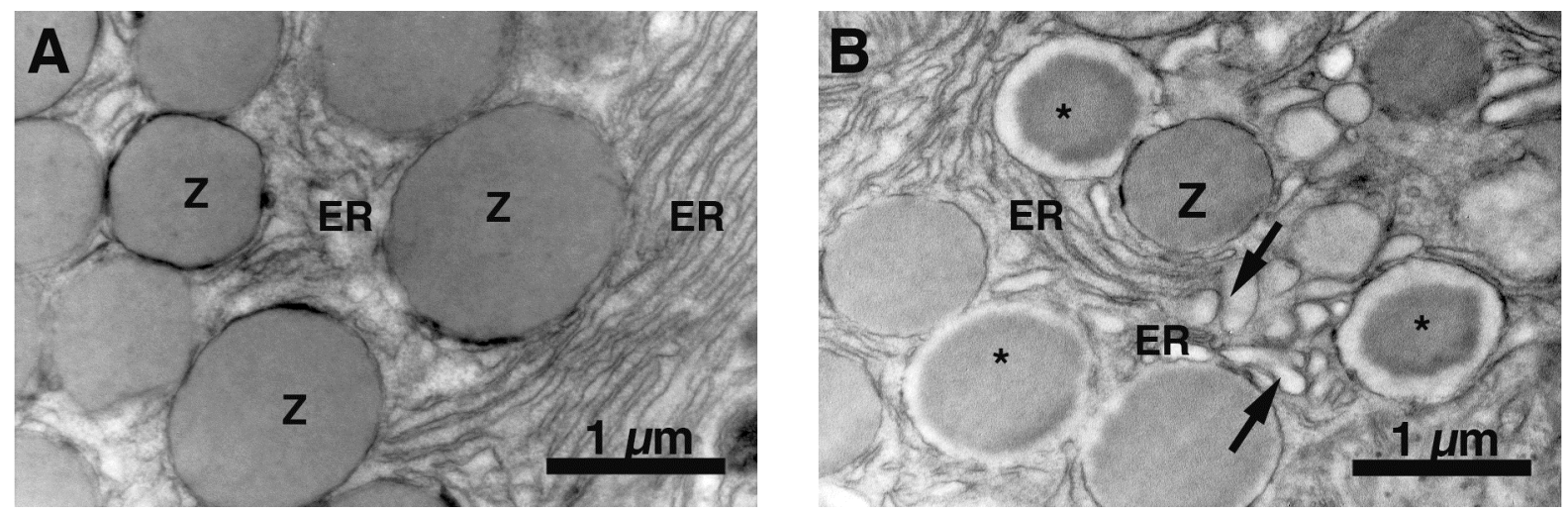

Fig 2. Pancreatic exocrine cells. (A) WT. (B) ERdj4 -/. In addition to ER dilation (arrow), immature zymogen granuels $(*)$ colocalized with electron dense zymogen granules in pancreatic exocrine cells of ERdj4 -/- mice. ER: endoplasmic reticulum; Z: zymogen granuels.
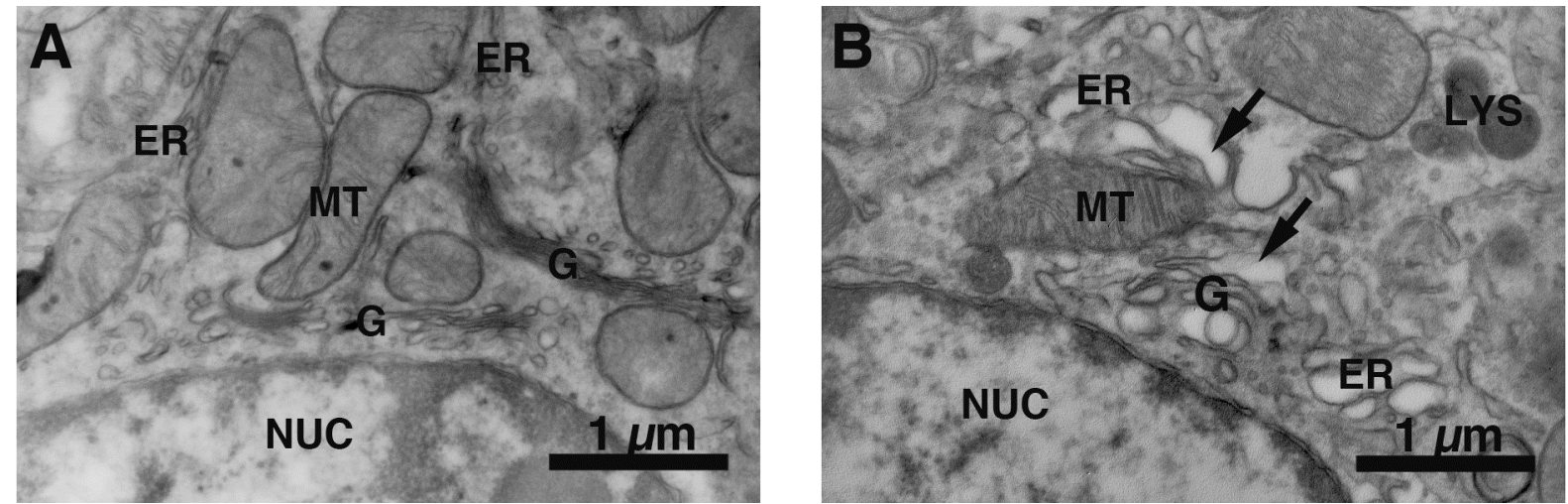

Fig 3. Murine epithelial cells of the convoluted tubules. (A) WT. (B) ERdj4 -/-. Dilated ER and inclusions (arrows) were detected in some ERdj4 -/- cells. ER: endoplasmic reticulum; G: Golgi complex; LYS:lysosome; MT: mitochondria; NUC: Nucleus. 